\title{
Promoter hypermethylation influences the suppressive role of maternally expressed 3, a long non-coding RNA, in the development of epithelial ovarian cancer
}

\author{
XIUJIE SHENG $^{1,3}$, JIANQI LI ${ }^{1,3}$, LEI YANG $^{2}$, ZHIYI CHEN $^{3}$, QIN ZHAO ${ }^{1}$, \\ LINYU TAN ${ }^{1}$, YANQING ZHOU ${ }^{1}$ and JUAN LI ${ }^{1}$
}

${ }^{1}$ Department of Obstetrics and Gynecology, Third Affiliated Hospital of Guangzhou Medical University, Guangzhou 510150;

${ }^{2}$ The Institute for Chemical Carcinogenesis, Guangzhou Medical University, Guangzhou 510182;

${ }^{3}$ Guangzhou Institute of Obstetrics and Gynecology, Guangzhou 510182, P.R. China

Received February 6, 2014; Accepted April 3, 2014

DOI: $10.3892 /$ or.2014.3208

\begin{abstract}
Maternally expressed 3 (MEG3) is a long noncoding RNA that can activate p53 and inhibit tumorigenesis and progression of various types of cancers. However, the role of MEG3 in epithelial ovarian cancer (EOC) is still unknown. The aim of the present study was to confirm whether MEG3 is downregulated in human EOC, determine its possible mechanism of action and elucidate the role of MEG3 in EOC. Differences in the expression of MEG3 and in the methylation status of the MEG3 promoter between EOC and normal ovary were analyzed using RT-PCR and methylation-specific PCR (MSP), respectively. MTT and EdU assays and flow cytometric analysis were used to assess the growth of ovarian cancer cells after overexpression of MEG3. The target genes regulated by MEG3 were detected with the Dual Luciferase Reporter system. The expression levels of target genes were confirmed using RT-PCR and western blotting. In contrast to normal ovarian tissues, the expression of MEG3 was absent or decreased in most EOC tissues as well as in human EOC cell lines, and the promoter of the MEG3 gene was highly methylated in both cancer tissues and cell lines. Treatment with 5-aza-2-deoxycytidine reversed the promoter hypermethylation and increased MEG3 expression. In addition, ectopic expression of MEG3 suppressed the proliferation and growth of OVCAR3 cells and promoted apoptosis. Finally, MEG3 activated p53 in OVCAR 3 cells. In conclusion, our data suggest that MEG3 is epigenetically silenced in EOC due to promoter hypermethylation, which may contribute to the development of EOC.
\end{abstract}

Correspondence to: Professor Xiujie Sheng, Department of Obstetrics and Gynecology, Third Affiliated Hospital of Guangzhou Medical University, Guangzhou 510150, P.R. China

E-mail: xjsheng@gzemail.cn

Key words: maternally expressed 3, long non-coding RNA, methylation, epithelial ovarian cancer

\section{Introduction}

Epithelial ovarian cancer (EOC) is one of the most common tumors of the female reproductive system, and the mortality rate of this disease ranks first among gynecological malignant tumors. In spite of continuous efforts to improve the therapeutic response, the prognosis for patients with EOC is still very poor, with an overall 5 -year survival of $\sim 50 \%$. Moreover, over $70 \%$ of patients with EOC suffer from relapse, although most advanced-stage patients undergo cytoreductive surgery and platinum-based chemotherapy (1).

Recently, several studies have revealed that long non-coding RNAs (lncRNAs), which have lengths of $>200$ nucleotides (nt), play important roles in tumor development (2). The maternally expressed 3 gene (MEG3) is an imprinted gene located on chromosome 14q32 that encodes an IncRNA-MEG3 RNA (3). MEG3 is expressed in many human tissues, such as ovary, testes and brain (4). However, recent studies have shown that expression of MEG3 is lost in multiple types of tumors including neuroblastomas, hepatocellular cancers and gliomas and in many cancer cell lines (5). Promoter methylation may account for the loss of expression of MEG3 in cancer. Studies have shown that promoter methylation plays a major role in silencing of the $M E G 3$ gene in clinically non-functioning adenomas (NFAs) (6), meningiomas (7) and neuroblastoma cell lines (8). Moreover, the results of Zhou et al (5) indicate that inactivation of the MEG3 gene in these tumors can be attributed in part to promoter silencing by hypermethylation.

MEG3 may act as a tumor suppressor during tumorigenesis. Overexpression of MEG3 inhibits the growth of cancer cell lines such as MCF7, HeLa (4), HCT116 and IOMM-Lee (9) and promotes apoptosis in HepG2, PLC/PRF/5, U251 and U87 MG cells $(10,11)$. Zhou et al determined that the antiproliferative activity of MEG3 was through regulation of tumor suppressor p53 (9).

However, the relationship between MEG3 and EOC has not been studied, and the role of MEG3 in EOC is still unknown. Therefore, on the basis of previous studies, we hypothesized that $M E G 3$ may be a novel tumor-suppressor gene in EOC. In the present study, we determined whether or not MEG3 RNA 
is downregulated in human EOC and inhibits EOC proliferation via targeting of p53.

\section{Materials and methods}

Tumor tissues and cell lines. Tissues from 20 normal ovaries and 20 EOCs were obtained from the Third Affiliated Hospital of Guangzhou Medical University and the General Hospital of Guangzhou Military Command of the People's Liberation Army (Table I) and frozen at $-80^{\circ} \mathrm{C}$. The histopathologic diagnoses were determined by the hospital's pathologist using International Federation of Gynecology and Obstetrics (FIGO) and morphologic criteria. Human ovarian cancer cell lines OVCAR3, SKOV3, HP8910 and ES-2 were purchased from the American Type Culture Collection (ATCC; Manassas, VA, USA), maintained in Dulbecco's modified Eagle's medium (DMEM) containing 10\% FBS (Gibco, Carlsbad, CA, USA) and cultured at $37^{\circ} \mathrm{C}$ with $5 \% \mathrm{CO}_{2}$. The present study was approved by the Institutional Review Board of Guangzhou Medical University.

RNA extraction and RT-PCR. Total RNA was extracted from 20 EOC and 20 normal ovarian tissue samples using RNAiso reagent (Takara, Japan). The reverse-transcription reactions were performed with PrimeScript RT Master Mix (Takara). PCR was performed using a Premix Taq version 2.0 (Takara) in accordance with the instructions from the respective manufacturer. The primers used to amplify MEG3 were as follows: 5'-GCC CTA GGG GAG TGA CTA CA (forward) and 5'-ACT CGG GAC ATA CCT GCT CT (reverse). The primers used to detect expression of $p 53, G D F 15$ and $R B 1$ were as follows: p53, 5'-CCT CAG CAT CTT ATC CGA GTG (forward) and 5'-TCA TAG GGC ACC ACC ACA C (reverse); GDF15, 5'-AAG AAC TCA GGA CGG TGA ATG (forward) and 5'-CCG CAA CTC TCG GAA TCT G (reverse); $R B 1$, 5'-GAA CTG TGG GGA ATC TGT ATC TTT (forward) and 5'-AAC TGC TGG GTT GTG TCA AAT A (reverse). $\beta$-actin was used as an internal reference. The reaction conditions were as follows: 30 cycles of $94^{\circ} \mathrm{C}$ for $5 \mathrm{~min}, 94^{\circ} \mathrm{C}$ for $30 \mathrm{sec}$, $58-60^{\circ} \mathrm{C}$ for $30 \mathrm{sec}$ and $72^{\circ} \mathrm{C}$ for $30 \mathrm{sec}$, and $1 \mathrm{cycle}$ of $72^{\circ} \mathrm{C}$ for $10 \mathrm{~min}$. The PCR products were resolved by electrophoresis through $2-3 \%$ agarose gels and visualized by ethidium bromide staining and the use of Semi-quantitative software (ImageJ). Each sample was analyzed in triplicate.

Methylation-specific PCR (MSP). DNA from human tissues was extracted from 20 EOC and 20 normal ovarian tissue samples using the QIAmp DNA Mini kit (Qiagen). Bisulfite treatment was performed with the EpiTect Bisulfite kit (Qiagen) in accordance with the manufacturer's instructions. The methylation status of the MEG3 gene was determined using conventional MSP. For the MSP, we used the EpiTect MSP kit (Qiagen). The sequences of the methylation-specific primers were as follows: the methylated pair (M), 5'-GTT AGT AAT CGG GTT TGT CGG C (forward) and 5'-AAT CAT AAC TCC GAA CAC CCG CG (reverse); the unmethylated pair (U), 5'-GAG GAT GGT TAG TTA TTG GGG T (forward) and 5'-CCA CCA TAA CCA ACA CCC TAT AAT CAC A (reverse). MEG3 DNA was amplified in a DNA Engine Peltier thermal cycler (Bio-Rad) by 1 cycle of $95^{\circ} \mathrm{C}$ for $15 \mathrm{~min}$ followed by 5 cycles of $94^{\circ} \mathrm{C}$ for $30 \mathrm{sec}, 70^{\circ} \mathrm{C}$ for $30 \mathrm{sec}$ and $72^{\circ} \mathrm{C}$ for $30 \mathrm{sec} ; 5$ cycles of $94^{\circ} \mathrm{C}$ for $30 \mathrm{sec}, 65^{\circ} \mathrm{C}$ for $30 \mathrm{sec}$ and $72^{\circ} \mathrm{C}$ for $30 \mathrm{sec} ; 30$ cycles of $94^{\circ} \mathrm{C}$ for $30 \mathrm{sec}, 60^{\circ} \mathrm{C}$ for $30 \mathrm{sec}$ and $72^{\circ} \mathrm{C}$ for $30 \mathrm{sec}$; and 1 cycle of $72^{\circ} \mathrm{C}$ for $7 \mathrm{~min}$. The PCR products were identified by electrophoresis through a $2.5 \%$ agarose gel and ethidium bromide staining (12).

Treatment with 5-aza-2-deoxycytidine. To explore the functional role of DNA methylation in the silencing of MEG3 transcription in ovarian cancer cells, OVCAR3, SKOV3, HP8910 and ES-2 cells were seeded onto 6-well plates at $1.0 \times 10^{5}$ cells/well and cultured in DMEM containing $1,5,10$ or $20 \mu \mathrm{M} 5$-aza-2-deoxycytidine (5-aza-CdR) (Sigma-Aldrich, USA) for 6 days $(6-8,10)$. Cells cultured in the absence of 5-aza-CdR were used as a control. The media, which contained different concentrations of 5-aza-CdR, were changed every day. RT-PCR and MSP were performed to measure the expression of MEG3 RNA and the methylation status of the MEG3 promoter, respectively, as described above.

Cell proliferation assay (MTT assay). To express MEG3, the $M E G 3$ cDNA was cloned into the pcDNA3.1 vector (referred to as pcDNA3.1-MEG3). The empty pcDNA3.1 vector (referred to as pcDNA3.1-empty) was used in the negative control (NC) group, and phosphate-buffered saline (PBS) was used in the blank group. Cellular proliferation was measured by the MTT assay. OVCAR3 cells were plated in 96-well plates at $\sim 5 \times 10^{3}$ cells/well in quintuplicate. After $\sim 12 \mathrm{~h}$ in culture, the cells were transfected with pcDNA3.1MEG3, pcDNA3.1-empty or PBS using Lipofectamine 2000 (Life Technologies, USA). The number of cells/well was determined by measuring the absorbance $(490 \mathrm{~nm})$ of MTT at 12, 24 and $48 \mathrm{~h}$ after transfection. Each experiment was repeated at least 3 times.

5-Ethynyl-2'-deoxyuridine (EdU) incorporation assay. OVCAR3 cells were plated in 24-well plates at $\sim 5.0 \times 10^{4}$ cells/well. The cells were transfected with pcDNA3.1MEG3, pcDNA3.1-empty or PBS when they reached $80 \%$ confluency. Forty-eight hours after transfection, EdU (50 mM) (Cell Light EdU DNA Imaging kit; Guangzhou RiboBio, China) was added, and the cells were cultured for an additional $2 \mathrm{~h}$. The cells were then stained with $100 \mathrm{mM}$ Apollo 567 fluorescent azide and Hoechst $(5 \mathrm{mg} / \mathrm{ml})$ for $30 \mathrm{~min}$. Images were acquired and analyzed using the High Content Imaging Pathway 855 (BD, USA), and the number of EdU-positive cells was calculated as follows: Number of EdU-positive cells/number of Hoechst-stained cells x $100 \%$ (13). Each experiment was repeated at least 3 times.

Flow cytometric analysis. For the cell cycle assay, $1.0 \times 10^{5}$ OVCAR3 cells were plated in 6-well plates and then transfected with pcDNA3.1-MEG3, pcDNA3.1-empty or PBS when they reached $80 \%$ confluencey. The cells were collected after $48 \mathrm{~h}$ and stained with propidium iodide (PI) and RNase (Cell Cycle Detection kit; KeyGene Biotech, China) to analyze the cell cycle with FACS (BD).

To assay apoptosis, OVCAR3 cells $\left(1.0 \times 10^{5} /\right.$ well) in 6-well plates were transfected with pcDNA3.1-MEG3, pcDNA3.1-empty or PBS. After 48 h, the cells were harvested, 
stained with Annexin V and PI (Annexin V-FITC Apoptosis Detection kit; KeyGene Biotech) in accordance with the manufacturer's protocol, and subjected to FACS analysis (BD Biosciences, USA).

Luciferase reporter assays. Since MEG3 has been reported to activate p53 by binding to the p53 promoter, we tested whether it can regulate the activity of p53 in EOC cells. OVCAR3 cells were plated in 6-well plates and transfected with pcDNA3.1-MEG3 and pGL3-p53 using Lipofectamine 2000. Forty-eight hours later, the cells were harvested, and luciferase activity was determined using the Dual-Luciferase kit (Promega, Madison, WI, USA) in accordance with the manufacturer's instructions. Luciferase activity was normalized to that of pRL-SV40 (Promega).

Western blotting. After being transfected with pcDNA3.1MEG3 or pcDNA3.1-empty, OVCAR3 cells were lysed with RIPA lysis buffer. Proteins were harvested and the concentrations of the samples were determined with the BCA protein assay kit (Beyotime, China). Then, the proteins were resolved on a $10 \%$ SDS denatured polyacrylamide gel and transferred to a PVDF membrane. After the membranes were blocked in $5 \%$ skim milk overnight at $4^{\circ} \mathrm{C}$, they were incubated with rabbit anti-human antibodies at the recommended dilution [p53, 1:500; GDF15, 1:700; RB1, 1:700 (all from BioWorld, USA)] and then incubated with the appropriate secondary antibody for $\sim 1 \mathrm{~h}$. Lab Works ${ }^{\mathrm{TM}}$ Image Acquisition and Analysis Software (UVP, USA) were used to quantify the intensities of the bands. GAPDH was used as a loading control.

Statistical analysis. All data are expressed as the means \pm SD from at least 3 separate experiments. Statistical differences were calculated using one-way ANOVA. The relationship between the extent of $\mathrm{CpG}$ methylation and expression of MEG3 RNA was assessed by association analysis. All calculations were performed with the software package GraphPad Prism 5.0. A probability (P)-value $<0.05$ was considered to indicate a statistically significant result.

\section{Results}

Downregulation of the IncRNA MEG3 in ovarian cancer. As shown in Fig. 1, the expression of MEG3 was downregulated in $25 \%$ (5 of 20) or absent in $70 \%$ (14 of 20) of the ovarian cancer tissues. In comparison, $85 \%$ (17 of 20) of the normal ovarian tissues expressed MEG3 at high levels. The difference in expression of MEG3 RNA between normal samples and cancer samples was significant $(\mathrm{P}<0.01)$. Moreover, as shown in Table I, MEG3 RNA was lost in 50\% (1 of 2) of grade I tumors, $75 \%$ (3 of 4 ) of grade II tumors and $71 \%$ (10 of 14) of grade III tumors. Furthermore, MEG3 RNA was not detected in any of the ovarian cancer cell lines (OVCAR3, SKOV3, HP8910 or ES-2; Fig. 3A).

Partial downregulation of MEG3 due to promoter methylation. An abnormal methylation pattern (M) of the MEG3 differentially methylated region (DMR) was observed in $60 \%$ (12 of 20) of patients with ovarian cancer who were evaluated. An unmethylated pattern (U) and a partially
Table I. Relationship between MEG3 RNA expression and promoter methylation status.

\begin{tabular}{|c|c|c|c|c|}
\hline Samples & $\begin{array}{c}\text { MEG3 RNA } \\
\text { level }\end{array}$ & Percent & $\begin{array}{l}\text { Promoter } \\
\text { status }\end{array}$ & Percent \\
\hline \multicolumn{5}{|l|}{ Normal } \\
\hline $\mathrm{N} 01^{\mathrm{a}}$ & High & $85 \%$ High & $\mathrm{U}$ & $0 \% \mathrm{M}$ \\
\hline N02 & High & $15 \%$ Low & $\mathrm{U}$ & $15 \% \mathrm{MU}$ \\
\hline N03 & High & & $\mathrm{U}$ & $85 \% \mathrm{U}$ \\
\hline N04 & High & & $\mathrm{U}$ & \\
\hline N05 & High & & $\mathrm{U}$ & \\
\hline N06 & High & & $\mathrm{U}$ & \\
\hline N07 & High & & $\mathrm{U}$ & \\
\hline N08 & High & & $\mathrm{U}$ & \\
\hline N09 & High & & $\mathrm{U}$ & \\
\hline N10 & Low & & MU & \\
\hline N11 & High & & $\mathrm{U}$ & \\
\hline N12 & High & & $\mathrm{U}$ & \\
\hline N13 & High & & $\mathrm{U}$ & \\
\hline N14 & Low & & MU & \\
\hline N15 & High & & $\mathrm{U}$ & \\
\hline N16 & High & & $\mathrm{U}$ & \\
\hline N17 & High & & $\mathrm{U}$ & \\
\hline N18 & Low & & MU & \\
\hline N19 & High & & $\mathrm{U}$ & \\
\hline $\mathrm{N} 20$ & High & & $\mathrm{U}$ & \\
\hline
\end{tabular}

Cancer

Grade I

$\begin{array}{ccccc}\text { C03 } & \text { Lost } & 50 \% \text { Lost } & \mathrm{M} & 50 \% \mathrm{M} \\ \text { C11 } & \text { High } & 50 \% \text { High } & \mathrm{U} & 50 \% \mathrm{U} \\ \text { Grade II } & & & & \\ \text { C04 } & \text { Lost } & 75 \% \text { Lost } & \mathrm{M} & 75 \% \mathrm{M} \\ \text { C06 } & \text { Low } & 25 \% \text { Low } & \mathrm{U} & 25 \% \mathrm{U} \\ \text { C10 } & \text { Lost } & & \mathrm{M} & \\ \text { C16 } & \text { Lost } & & \mathrm{M} & \end{array}$

Grade III

$\mathrm{C} 01$

Low

$71.4 \%$ Lost U

$57.2 \% \mathrm{M}$

$\mathrm{C} 02$

Lost

$28.6 \%$ Low

$\mathrm{M}$

$35.7 \% \mathrm{MU}$

$\mathrm{C} 05$

Low

$\mathrm{C} 07$

Lost

MU

$7.1 \% \mathrm{U}$

$\mathrm{C} 08$

Lost

M

MU

C09 Lost

$\mathrm{M}$

$\mathrm{M}$

M

C13 Lost

MU

C14 Lost

C15 Low

MU

C17 Lost

$\mathrm{M}$

$\mathrm{M}$

$\mathrm{M}$

C19

Lost

MU

${ }^{a} \mathrm{~N}$, normal sample; $\mathrm{C}$, cancer sample; $\mathrm{M}$, hypermethylation; $\mathrm{U}$, unmethylation; MU, partial methylation; MEG3, maternally expressed 3 . 


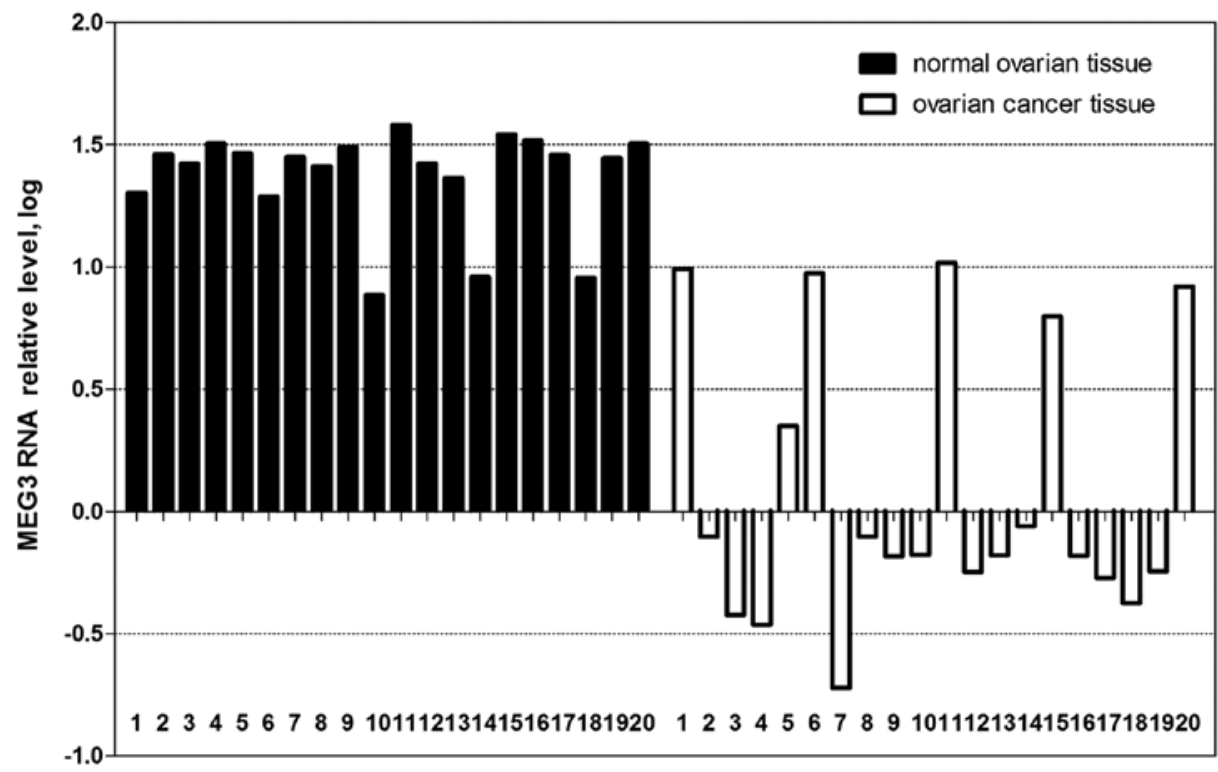

Figure 1. Downregulation of expression of the long non-coding RNA MEG3 in EOC. RT-PCR was used to measure the expression of MEG3 RNA in 20 epithelial ovarian cancer samples and 20 normal ovarian tissue samples. The relative expression is presented as the fold-change on a log scale. Samples were classified as 'high-expression' if the lower limit of the log value was $>1$, as 'low-expression' if the upper limit was $<1$ but $>0$, and as 'lost expression' if the upper limit was <0 (14). Compared with the normal samples, of which 85\% (17 of 20) were 'high-expression', 5\% (1 of 20) of the cancer samples were 'high-expression', 25\% (5 of 20) were 'low-expression' and 70\% (14 of 20) were 'lost expression' ( $\mathrm{P}<0.01$ ). MEG3, maternally expressed 3; EOC, epithelial ovarian cancer.

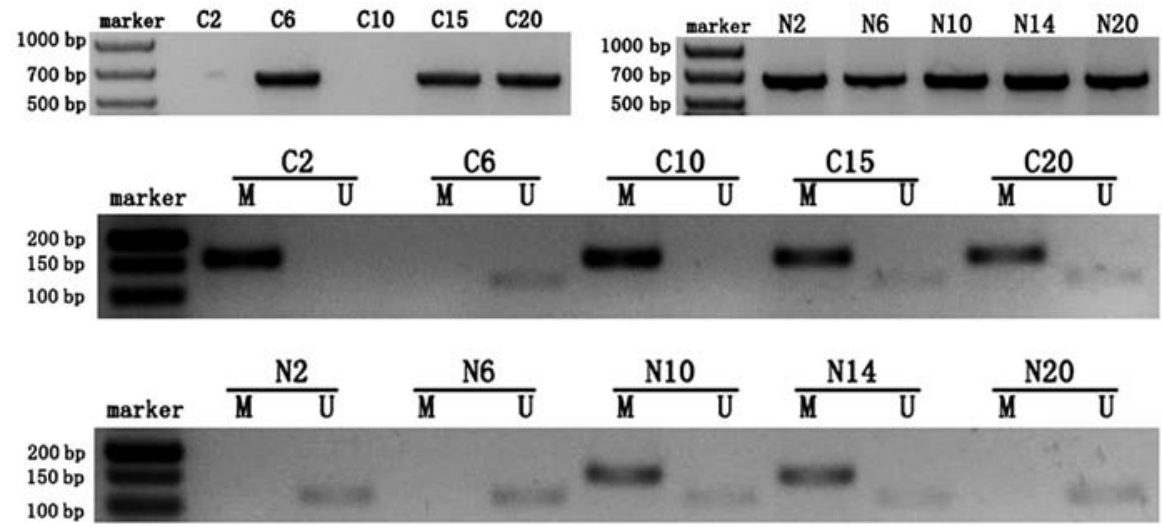

Figure 2. Expression of MEG3 RNA and methylation status of the $M E G 3$ promoter in ovarian cancer. $\mathrm{C} 2, \mathrm{C} 6, \mathrm{C} 10, \mathrm{C} 15$ and $\mathrm{C} 20$ represent ovarian cancer tissue samples; N2, N6, N10, N14 and N20 represent normal ovarian tissue samples. The size of the MEG3 product was 659 bp. The sizes of the bands for the hypermethylated pattern (M) and the unmethylated pattern (U) were 160 and 120 bp, respectively. MEG3, maternally expressed 3.

methylated pattern ( $\mathrm{M}$ and $\mathrm{U})$ were observed in 15 and $25 \%$, respectively, of the 20 cancer samples. In comparison, most of the normal ovarian tissue samples $(85 \%, 17$ of 20$)$ displayed an unmethylated pattern (U). The difference in MEG3 expression between normal and cancer samples was significant $(\mathrm{P}<0.01)$ after using the Chi-square test to correct for continuity. As shown in Fig. 2 and Table I, all of the ovarian cancer samples that were categorized as 'lost expression' displayed a hypermethylated pattern (M) while all of the normal samples that were categorized as 'high-expression' displayed an unmethylated pattern (U). Furthermore, the MEG3 promoter was hypermethylated in 50\% (1 of 2) of grade I tumors, 75\% (3 of 4 ) of grade II tumors and $57 \%$ (8 of 14) of grade III tumors. Association analysis indicated that a significant negative correlation existed between the extent of CpG methylation and the expression of MEG3 RNA $\left(\chi^{2}=32.73, r=-0.905, P<0.01\right)$. Methylation in the 4 ovarian cancer cell lines (OVCAR3, SKOV3, HP8910 and ES-2) was detected by MSP. This analysis revealed that all 4 of the cell lines were abnormally methylated (M; Fig. 3A). Collectively, the data illustrate that partial downregulation of MEG3 was due to hypermethylation of the $M E G 3$ promoter and that the methylation status correlates with EOC grade.

Expression of MEG3 RNA resumes after treatment with 5-aza-CdR. We treated human ovarian cancer OVCAR3 cells with different concentrations of 5-aza-CdR. As shown in Fig. 3B-D, treatment with 1, 5, 10 or $20 \mu \mathrm{M}$ of 5 -aza-CdR resulted in re-expression of MEG3 and partially reversed the abnormal methylation pattern of the MEG3 promoter. These 
A
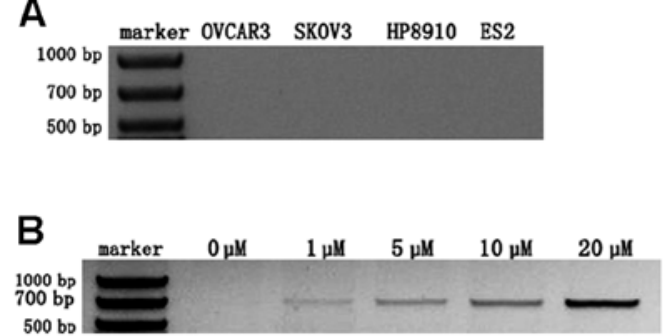

C

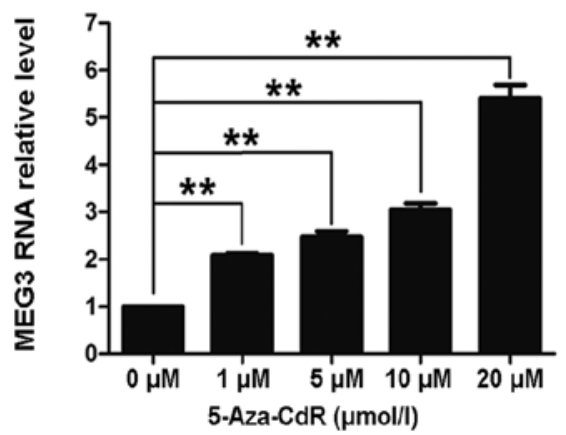

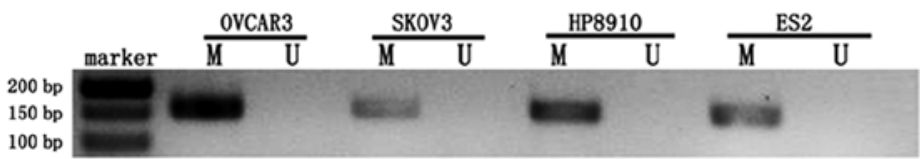

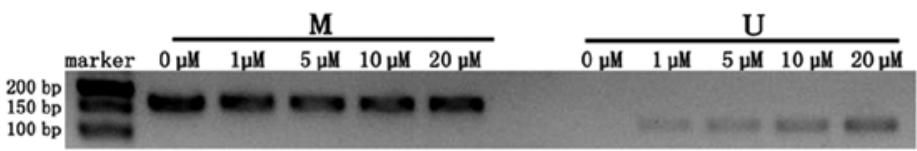

D

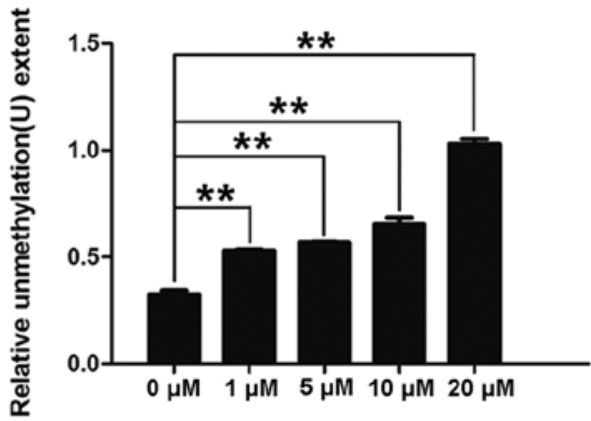

Figure 3. Expression of MEG3 RNA and methylation status of the MEG3 promoter in ovarian cancer cell lines with or without treatment with 5-aza-2-deoxycytidine (5-aza-CdR). (A) MEG3 expression was lost and the MEG3 promoter was abnormally methylated (M) in OVCAR3, SKOV3, HP8910 and ES-2 cells. (B) MEG3 RNA was re-expressed in OVCAR3 cells treated with different concentrations of 5-aza-CdR (1,5,10 and $20 \mu \mathrm{M})$, and compared to cells of the control group $(0 \mu \mathrm{M} 5$-aza-CdR), cells of the 1,5, 10 and $20 \mu \mathrm{M}$ 5-aza-CdR groups displayed less CpG methylation. (C) The level of MEG3 increased with increasing concentrations of 5-aza-CdR $\left(^{* *} \mathrm{P}<0.01\right)$. (D) The U level increased with the increasing concentrations of 5-aza-CdR (*** $\left.\mathrm{P}<0.01\right)$. MEG3, maternally expressed 3 .

results indicate that $\mathrm{CpG}$ methylation may lead to downregulation of MEG3 RNA in ovarian cancer cell lines.

MEG3 inhibits proliferation and promotes apoptosis of ovarian cancer cells. We used RT-PCR to determine that MEG3 RNA was re-expressed in OVCAR3 cells after they were transfected with pcDNA3.1-MEG3. MEG3 RNA was not detected in cells of the NC and blank groups (Fig. 4A). The difference in the expression of MEG3 between the MEG3 group and the NC and blank groups was significant $(\mathrm{P}<0.01)$.

To investigate the role of MEG3 in cell growth, OVCAR3 cells were transfected with pcDNA3.1-MEG3 and then subjected to proliferation and apoptosis assays. Results of the MTT assay showed that proliferation was significantly inhibited in cells that overexpressed MEG3. In contrast to the NC and blank groups, the inhibition ratio of the MEG3 group at 12,24 and $48 \mathrm{~h}$ after transfection was significantly different (37.26, 38.19 and 39.73\%, respectively; $\mathrm{P}<0.01$; Fig. 5A). An EdU assay showed that the percentage of cells in the $\mathrm{S}$ phase was reduced by $35.01 \pm 2.68 \%$ in OVCAR3 cells that overexpress MEG3 ( $\mathrm{P}<0.01$; Fig. 5B). The outcome of analysis of the cell cycle indicated that OVCAR3 cells were arrested at the G0/G1 phase and that the percentages of cells in the $\mathrm{S}$ and G2/M phases were also decreased (Fig. 5C). An apoptosis assay showed that overexpression of MEG3 caused OVCAR3 cells to undergo apoptosis (Fig. 5D). All of the data presented above support the hypothesis that MEG3 negatively regulates the growth of ovarian cancer cells.

Association of MEG3 and p53. To understand the molecular mechanism by which MEG3 suppresses the growth of ovarian
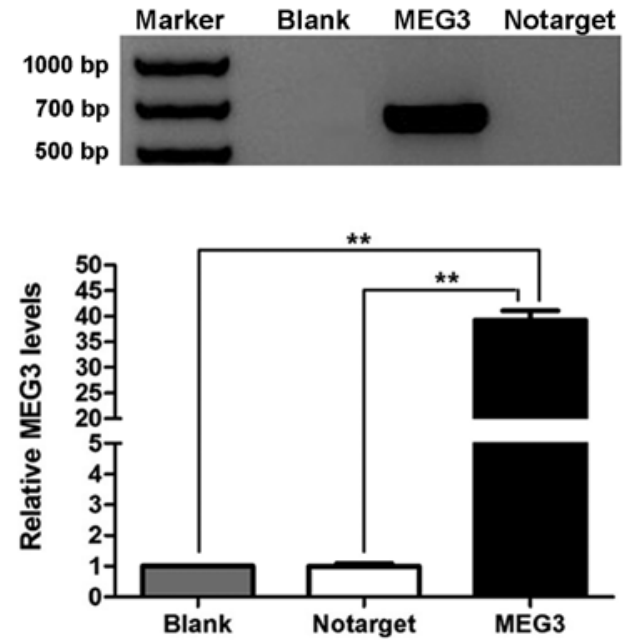

Figure 4. OVCAR3 cells re-express MEG3 RNA after transfection with a MEG3 expression vector. Compared to cells of the NC and blank groups, the level of expression of MEG3 RNA was significantly greater in cells of the MEG3 group $\left({ }^{* *} \mathrm{P}<0.01\right)$. MEG3, maternally expressed 3; NC, negative control. Notarget represents the negative control group.

cancer cells, we investigated whether MEG3 regulates the activation of p53. OVCAR3 cells were cotransfected with pGL3-p53 and pcDNA3.1-MEG3. Fig. 6A shows that cells transfected with pcDNA3.1-MEG3 had significantly increased levels of p53 activity. Additionally, we also found that overexpression of MEG3 in OVCAR3 cells increased p53, GDF15 and $R B 1$ mRNA and protein levels (Fig. 6B and C). 
A

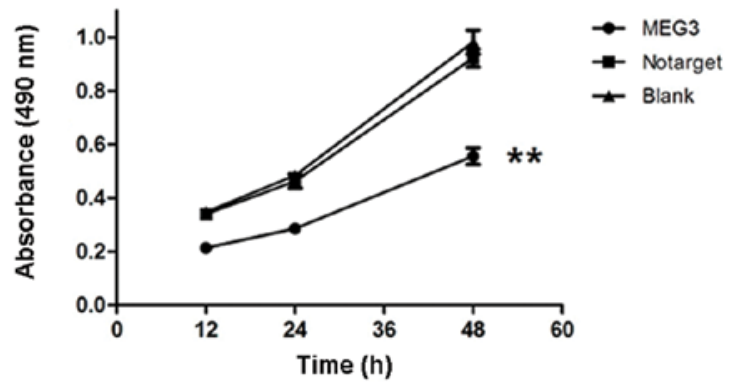

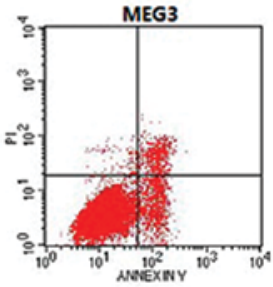

$5.75 \pm 0.25$

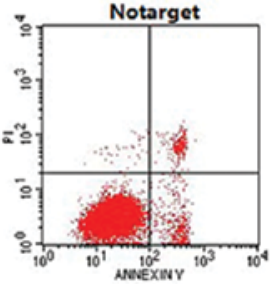

$1.60 \pm 0.17$

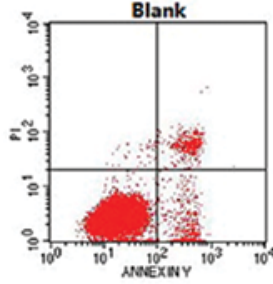

$1.73 \pm 0.14$

B
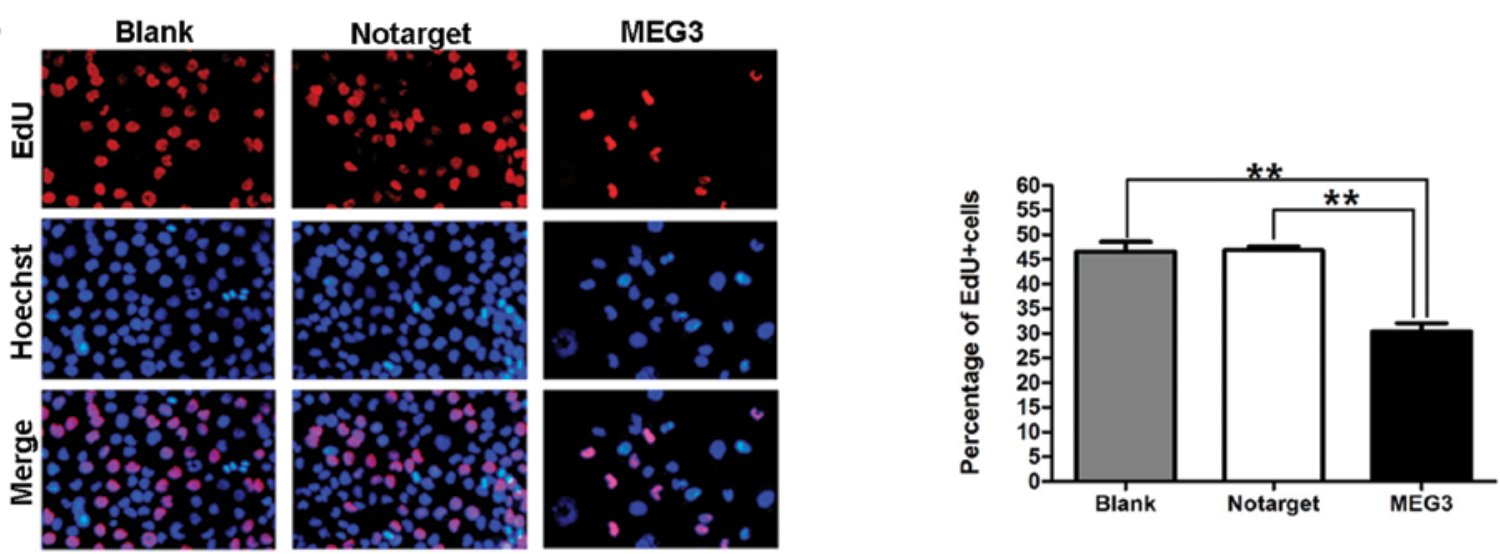

C
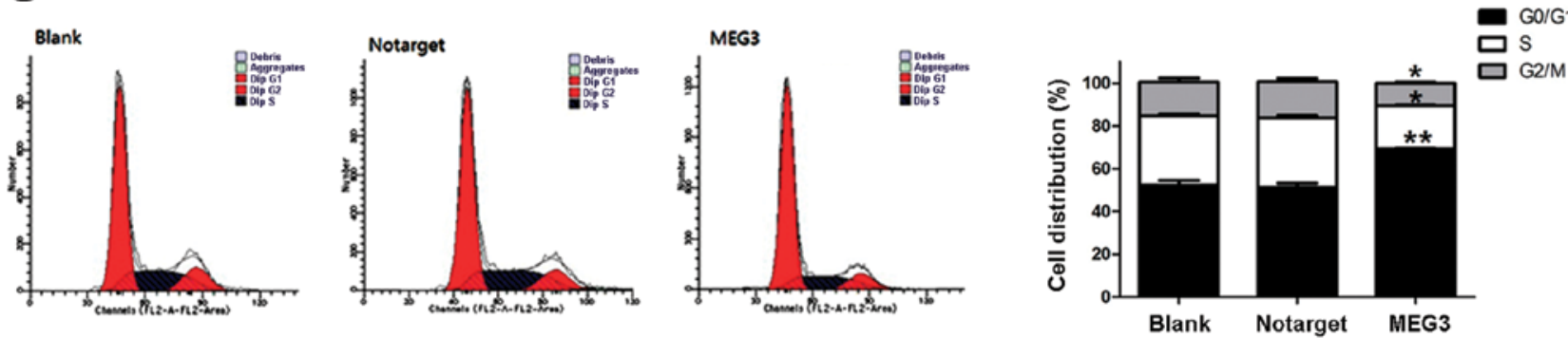

Figure 5. Exogenous expression of MEG3 affects proliferation and apoptosis of ovarian cancer cells. (A) OVCAR3 cells were transfected with pcDNA3.1-MEG3, and the number of cells/well was determined 12, 24 and $48 \mathrm{~h}$ later by measuring the absorbance $(490 \mathrm{~nm})$ of MTT. The data are from at least 3 independent experiments and are expressed as the means $\pm \mathrm{SD}\left({ }^{* *} \mathrm{P}<0.01\right)$. (B) An EdU assay of the relative number of Hoechst-stained cells and EdU-positive cells. OVCAR3 cells were transfected with pcDNA3.1-MEG3, pcDNA3.1-empty or phosphate-buffered saline (PBS). Forty-eight hours after the transfections, EdU $(50 \mathrm{mM})$ was added to the media, and the cells were cultured for $2 \mathrm{~h}$. EdU and Hoechst staining were performed as described in Materials and methods. (C) Analysis of the cell cycle of OVCAR3 cells overexpressing MEG3 and control OVCAR3 cells. The cells were harvested $48 \mathrm{~h}$ after transfection with pcDNA3.1-MEG3 or pcDNA3.1-empty and then stained with propidium iodide (PI). The cell cycle was analyzed with FACS. The data shown are the averages of at least 3 independent runs, compared with data from the negative control $(\mathrm{NC})$ and blank groups $\left({ }^{* *} \mathrm{P}<0.01,{ }^{*} \mathrm{P}<0.05\right)$. (D) OVCAR3 cells were transfected with pcDNA3.1-MEG3 and then apoptosis was detected using flow cytometry. Overexpression of MEG3 promoted apoptosis in OVCAR3 cells. Notarget represents the negative control group. MEG3, maternally expressed 3; EdU, 5-ethynyl-2'-deoxyuridin.

\section{Discussion}

We determined that MEG3 RNA is highly expressed in most normal human ovarian tissues. However, expression of MEG3 in epithelial ovarian cancers (EOCs) was sharply decreased and sometimes even lost. Expression of MEG3 RNA was not detected in $>70 \%$ of the EOC samples, and this loss of expression was associated with the grade of the tumor. We also discovered that MEG3 RNA was not expressed in the OVCAR3, SKOV3, HP8910 and ES-2 cell lines. On the other hand, the $M E G 3$ promoter was hypermethylated in $>60 \%$ of the EOC samples and in $100 \%$ of the ovarian cancer cell lines. As was determined in previous reports, the demethylating agent 5-aza-CdR reversed the hypermethylation of the MEG3 promoter in OVCAR3 cells, with the effectiveness increasing with increasing concentrations of the drug.

Is there a relationship between downregulation or loss of expression of MEG3 RNA and hypermethylation of the MEG3 promoter?

MEG3 is a long non-coding RNA (lncRNA). The MEG3 gene, also known as gene trap locus 2 ( $g t l 2)$, is located on human chromosome 14q32 (Meg3 is located on mouse chromosome 12) and is reciprocally imprinted with the paternally expressed gene DLK1 (15). Two DMRs are located upstream of the MEG3 gene, IG-DMR and MEG3-DMR, which controls expression in the placenta and the body, respectively (16). 

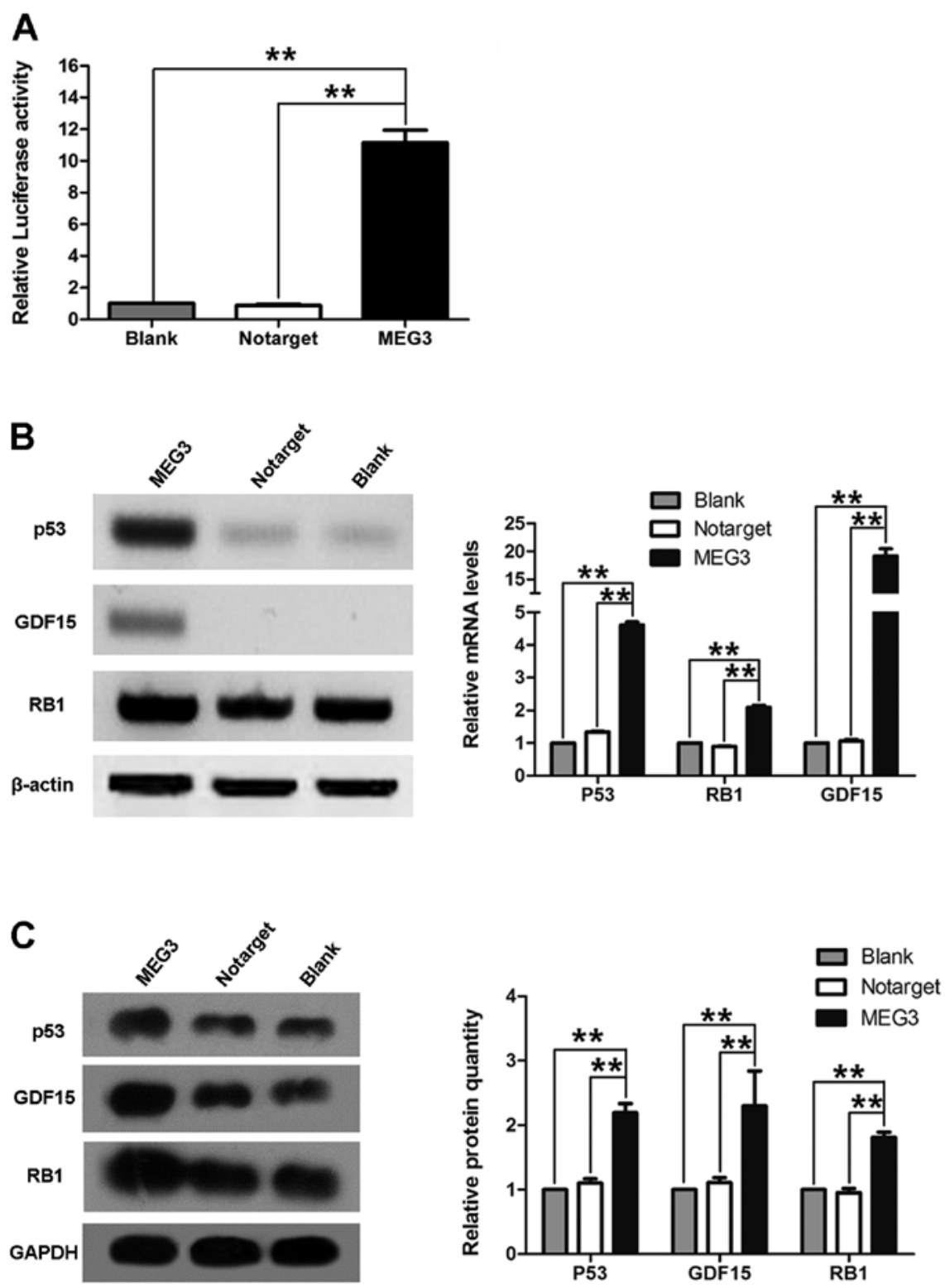

Figure 6. (A) Luciferase activity of p53 in OVCAR3 cells transfected with pcDNA3.1-MEG3, pcDNA3.1-empty or phosphate-buffered saline (PBS). The results revealed that activity of the p53 promoter in OVCAR3 cells overexpressing MEG3 was elevated by $11.14 \pm 0.46-$ fold (** $\mathrm{P}<0.01$ ). (B) $p 53$, GDF15 and $R B 1$ mRNA levels were increased after overexpression of MEG3 in OVCAR3 cells ( $\left.{ }^{* * *} \mathrm{P}<0.01\right)$. (C) Protein was extracted from OVCAR3 cells transfected with either pcDNA3.1-MEG3 or pcDNA3.1-empty. Compared with levels of p53, RB1 and GDF15 in cells of the NC group, levels in cells of the MEG3 group were increased $\left({ }^{* *} \mathrm{P}<0.01\right)$. MEG3, maternally expressed 3 . Notarget represents the negative control group.

Zhao et al used bisulfite sequencing to determine that the methylation in regions 1 and 4 of the MEG3 promoter is significantly higher in NFAs than in normal pituitary and that the MEG3 promoter overlaps with the MEG3-DMR (6). Hypermethylation of the MEG3 promoter is also observed in meningiomas (7) and neuroblastoma cell lines (8). Downregulation or loss of expression of MEG3 RNA is consistent with abnormal methylation of the $M E G 3$ promoter in these types of cancers. In contrast, MEG3 is expressed in many normal human tissues whose promoters do not display $\mathrm{CpG}$ hypermethylation. These studies indicate that inactivation of the MEG3 gene and downregulation of MEG3 RNA in these tumors partially result from silencing of the promoter by hypermethylation (5). Moreover, treating cells with 5 -azaCdR triggers re-expression of MEG3 in multiple human breast cancer tumor cell lines (6), meningiomas (7), neuroblastomas (8) and hepatocellular carcinomas (10).

Epigenetic silencing of tumor-suppressor genes by methylation is now arousing more and more interest in cancer research. DNA methylation is an epigenetic modification that is important in transcriptional control (17). Hypermethylation of the promoters of these genes is associated with gene silencing. Similar to gene mutation, epigenetic silencing is another mechanism by which tumor-suppressor genes are inactivated (18). Epigenetic changes have been implicated in malignant transformation and progression of different types of cancers, including ovarian cancer (18). For example, the RASSF $1 A$ gene is methylated in $\sim 40-50 \%$ of ovarian cancers $(19,20)$. Moreover, hypermethylation of the BRCAI promoter is highly correlated with decreased expression of 
BRCA1 and BRCA2 mRNA and is significantly correlated with tumor stage (21).

Our findings are consistent with those of the studies mentioned above and strongly indicate that hypermethylation of the $M E G 3$ promoter plays a significant role in inactivation of the $M E G 3$ gene in EOC.

Functionally, in a colony-formation assay, overexpression of MEG3 in MCF7 and HeLa cells was found to result in a significantly lower number of colonies in these cells than in cells transfected with the controls (4). In addition, ectopic expression of MEG3 significantly inhibited the incorporation of BrdU in HCT116 and IOMM-Lee cells (15). Braconi et al (10) and Wang et al (11) reported that ectopic expression of MEG3 caused apoptosis in PLC/PRF/5 and U251 and U87 MG cells, respectively. We proposed that MEG3 may play the same role in EOC. The results of the present study indicated that overexpression of MEG3 inhibited proliferation and promoted apoptosis in ovarian cancer cells. Downregulation of MEG3 in ovarian cancer cells appears to enhance the growth of the neoplasm.

In general, the tumor suppressor p53 potently inhibits cell growth by blocking proliferation or by activating cell death programs (22). Recent studies have demonstrated that lncRNAs physically associate with p53, suggesting that they may play an important role in regulating cell growth via the p53 pathway (23). Normally, the level of p53 is very low as the ubiquitin-proteasome system induces its rapid degradation. MDM2 is a member of this system, which inhibits p53 function and promotes p53 protein degradation (24). MEG3 targets p53 by either directly interacting with p53 or indirectly suppressing the negative regulator MDM2 (9). These effects may result in selective activation of downstream targets of p53 such as GDF15 and other yet-to-be-identified proteins with antiproliferative and tumor-suppressive functions (5). The RB pathway may be the other mechanism by which MEG3, independently of p53, functions as a tumor suppressor (9). Transfecting HCT116 cells with an MEG3 expression vector induces a significant increase in p53 (9). In the present study, $p 53$ mRNA and protein levels and the activity of the $p 53$ promoter were increased in OVCAR3 cells transfected with pcDNA3.1-MEG3. Moreover, in the present study, expression of GDF15 and RBI was 2-fold greater than that in control cells. These data indicate that MEG3 targets p53 to exert an antiproliferative function.

Although Braconi et al (10) determined that microRNA-29 can regulate the expression of MEG3 in hepatocellular cancer by modulating DNA methyltransferase (DNMT) 1 and 3b, the mechanisms upstream of DNMT regulation of MEG3 need to be identified. Staub et al (25) found that HSULF1 is epigenetically silenced in ovarian cancer and that epigenetic therapy targeting HSULF1 rendered ovarian tumors sensitive to conventional firstline therapies. Our future studies will confirm whether or not targeting MEG3 with 5-aza-2-deoxycytidine treatment may sensitize EOC to chemotherapy.

In conclusion, our data suggest that MEG3 may play an important role as a tumor suppressor in ovarian cancer cells since: i) $M E G 3$ is located at the chromosome $14 \mathrm{q} 32$ locus; ii) MEG3 is highly expressed in normal ovarian tissues, but its expression is decreased or absent in EOC tissues and ovarian cancer cells; iii) hypermethylation of the $M E G 3$ promoter is negatively correlated with the expression of MEG3 RNA in EOCs; iv) re-expression of MEG3 in ovarian cancer cells strongly suppresses growth and promotes apoptosis; and finally v) MEG3 indirectly or directly targets p53 and/or RB1 to control proliferation of ovarian cancer cells (15). Further investigation of the mechanism of action of MEG3 could therefore possibly provide new therapeutic strategies for EOC.

\section{Acknowledgements}

We thank Qicai Liu and Jiachun Lu for the helpful advice with experiments and careful reading of this manuscript. This study was supported by grants from the Science and Information Technology Agency of Guangzhou (no. 2010GNE00221).

\section{References}

1. Itamochi H: Targeted therapies in epithelial ovarian cancer: molecular mechanisms of action. World J Biol Chem 1: 209-220, 2010.

2. Gibb EA, Brown CJ and Lam WL: The functional role of long non-coding RNA in human carcinomas. Mol Cancer 10: 38, 2011.

3. Miyoshi N, Wagatsuma H, Wakana S, et al: Identification of an imprinted gene, Meg3/Gtl2 and its human homologue MEG3, first mapped on mouse distal chromosome 12 and human chromosome 14q. Genes Cell 5: 211-220, 2000.

4. Zhang X, Zhou Y, Mehta KR, et al: A pituitary-derived MEG3 isoform functions as a growth suppressor in tumor cells. J Clin Endocrinol Metab 88: 5119-5126, 2003.

5. Zhou Y, Zhang X and Klibanski A: MEG3 noncoding RNA: a tumor suppressor. J Mol Endocrinol 48: R45-R53, 2012.

6. Zhao J, Dahle D, Zhou Y, Zhang X and Klibanski A: Hypermethylation of the promoter region is associated with the loss of $M E G 3$ gene expression in human pituitary tumors. J Clin Endocrinol Metab 90: 2179-2186, 2005.

7. Zhang X, Gejman R, Mahta A, et al: Maternally expressed gene 3, an imprinted noncoding RNA gene, is associated with meningioma pathogenesis and progression. Cancer Res 70: 2350-2358, 2010.

8. Astuti D, Latif F, Wagner K, et al: Epigenetic alteration at the DLK1-GTL2 imprinted domain in human neoplasia: analysis of neuroblastoma, phaeochromocytoma and Wilms' tumour. Br J Cancer 92: 1574-1580, 2005.

9. Zhou Y, Zhong Y, Wang Y, et al: Activation of p53 by MEG3 non-coding RNA. J Biol Chem 282: 24731-24742, 2007.

10. Braconi C, Kogure T, Valeri N, et al: microRNA-29 can regulate expression of the long non-coding RNA gene MEG3 in hepatocellular cancer. Oncogene 30: 4750-4756, 2011.

11. Wang $\mathrm{P}$, Ren $\mathrm{Z}$ and Sun $\mathrm{P}$ : Overexpression of the long non-coding RNA MEG3 impairs in vitro glioma cell proliferation. J Cell Biochem 113: 1868-1874, 2012.

12. Benetatos L, Hatzimichael E, Dasoula A, et al: CpG methylation analysis of the MEG3 and SNRPN imprinted genes in acute myeloid leukemia and myelodysplastic syndromes. Leuk Res 34: 148-153, 2010.

13. Feng S, Cong S, Zhang X, et al: MicroRNA-192 targeting retinoblastoma 1 inhibits cell proliferation and induces cell apoptosis in lung cancer cells. Nucleic Acids Res 39: 6669-6678, 2011.

14. Anwar SL, Krech T, Hasemeier B, et al: Loss of imprinting and allelic switching at the DLK1-MEG3 locus in human hepatocellular carcinoma. PLoS One 7: e49462, 2012.

15. Benetatos L, Vartholomatos G and Hatzimichael E: MEG3 imprinted gene contribution in tumorigenesis. Int J Cancer 129: 773-779, 2011.

16. Kagami M, O'Sullivan MJ, Green AJ, et al: The IG-DMR and the $M E G 3$-DMR at human chromosome 14q32.2: hierarchical interaction and distinct functional properties as imprinting control centers. PLoS Genet 6: e1000992, 2010.

17. Jones PA and Baylin SB: The fundamental role of epigenetic events in cancer. Nat Rev Genet 3: 415-428, 2002. 
18. Baylin SB and Ohm JE: Epigenetic gene silencing in cancer - a mechanism for early oncogenic pathway addiction? Nat Rev Cancer 6: 107-116, 2006.

19. Yoon JH, Dammann R and Pfeifer GP: Hypermethylation of the $\mathrm{CpG}$ island of the RASSF1A gene in ovarian and renal cell carcinomas. Int J Cancer 94: 212-217, 2001.

20. Ibanez de Caceres I, Battagli C, Esteller M, et al: Tumor cell-specific BRCAI and RASSF1A hypermethylation in serum, plasma, and peritoneal fluid from ovarian cancer patients. Cancer Res 64: 6476-6481, 2004

21. Chan KY, Ozçelik H, Cheung AN, Ngan HY and Khoo US: Epigenetic factors controlling the $B R C A 1$ and $B R C A 2$ genes in sporadic ovarian cancer. Cancer Res 62: 4151-4156, 2002
22. Kim T, Veronese A, Pichiorri F, et al: p53 regulates epithelialmesenchymal transition through microRNAs targeting ZEB1 and ZEB2. J Exp Med 208: 875-883, 2011.

23. Huarte M, Guttman M, Feldser D, et al: A large intergenic noncoding RNA induced by 53 mediates global gene repression in the p53 response. Cell 142: 409-419, 2010.

24. Brooks $\mathrm{CL}$ and $\mathrm{Gu} \mathrm{W}: \mathrm{p} 53$ regulation by ubiquitin. FEBS Lett 585: 2803-2809, 2011.

25. Staub J, Chien J, Pan Y, et al: Epigenetic silencing of HSulf-1 in ovarian cancer:implications in chemoresistance. Oncogene 26 : 4969-4978, 2007. 